\title{
Using Bayesian Network for Monetary Structure and Supply Researching
}

\author{
Zhang $X u^{1, a}$, Zhu Ruihai ${ }^{2, b}$ and Luo Jun ${ }^{3, c}$ \\ ${ }^{1}$ Yangtze University Economic School, China \\ apayi.zx@gmail.com, b499314978@qq.com, c374486904@qq.com
}

Keywords: Monetary Supply, Monetary Structure, Bayesian Network

\begin{abstract}
In this paper, we use Bayesian network structure learning methods, $K 2$ and $M C M C$, to build a monetary network and for making the data more visible we also use Gephi to draw the net. In the end, we get 174 nodes and 1443 lines about the macroeconomic targets and find the main factors has influence on monetary structure and supplement.
\end{abstract}

\section{Introduction}

As Keynes said in <The general theory of employment, interest and money> "If, however, we are tempted to assert that money is the drink which stimulates the system to activity" ${ }^{[1]}$ and "We shall discover, however, that Money plays an essential part in our theory of the Rate of Interest; and we shall attempt to disentangle the peculiar characteristics of Money which distinguish it from other things. “[2]

Macro finance is a subject to reveal the monetary working rules. The first step of revealing is to calculate the monetary supplyment that are M0, M1, and M2,in China, also the basic targets for the macroeconomic and monetary policy.

But, actually, the whole economy has so many sophisticated connections with other things such like trade, finance and education, etc, that even a tiny part of them can have a huge influence on the virtual economy. Thus from our view of point, we regard the connections as a network. To build and find its characteristics about each nodes can tell us the objective relationships of the whole economy.

\section{Bayesian Network}

Probability is the base of Bayesian network and that is why Bayesian network designed to solve how to calculate the joint probability. In this algorithm, people use probability calculating formulas to deduce posterior probability and build an artificial net.

\section{Definition of Bayesian Network.}

1) Total probability formula

A case can be defined like that: $E$ is a case, and which obey to 1$) U_{i=1}^{n} B_{i}=\Omega$ ( $\Omega$ is sample space) (2) $B_{i} B_{j}=\phi, i \neq j$, so we call this: $p(A)=\sum_{i=1}^{n} p\left(B_{i}\right) p\left(A \mid B_{i}\right)$ as total probability formula.The discrete form goes like this: $p(Y=y)=\sum_{i=1}^{n} p\left(X_{i}=x_{i}\right) p\left(Y=y \mid X_{i}=x_{i}\right)$ the continuous variable form is: $p(y)=\int p(y \mid x) p(x) d x$

2) Bayesian formula

Also we define $B_{1}, B_{2}, \ldots, B_{n}$ as a incompatible case group of $E$, and $p\left(B_{i}\right)>0, A$ is an arbitrarily case of $E$, so:

$$
\begin{gathered}
P\left(B_{i} \mid A\right)=\frac{p\left(B_{i}\right) p\left(A \mid B_{i}\right)}{\sum_{j=1}^{n} p\left(B_{j}\right) p\left(A \mid B_{j}\right)} \text { is Bayesian formula. The discrete form goes like this: } \\
P\left(X_{i}=x_{i} \mid Y=y\right)=\frac{p \sum_{i=1}^{n} p\left(X_{i}=x_{i}\right) p\left(Y=y \mid X_{i}=x_{i}\right)}{\sum_{j=1}^{n} p\left(X_{j}=x_{j}\right) p\left(Y=y \mid X_{j}=x_{j}\right)}
\end{gathered}
$$

The continuous variable form:

Firstly, we assume the joint distribution density of random variables $\xi$ and $\eta$ are $p(x, y)=p_{\xi}(x) f_{\eta \mid \xi}(y \mid x)$ the $p_{\xi}(x)$ is edge density of $\xi$, and $f_{\eta \mid \xi}(y \mid x)$ is the conditional density when $\xi=x$. So the form can be expressed like this: 
Bayesian Network Structure Learning Method.

$$
g_{\xi \mid \eta}(x \mid y)=\frac{p(x) f_{\eta \mid \xi}(y \mid x)}{\int p_{\xi}(x) f_{\eta \mid \xi}(y \mid x) d x}
$$

In Bayesian structure learning method we can find a reasonable structure of each nodes distribution by some testing and inferring methods. In our paper we decide use K2 and MCMC methods.

1) The Score Standard of Structure in Bayesian Network (K2)

For known data group $D, K 2$ method use the posterior probability $p\left(G_{B} \mid D\right)$ to find the best structure. And for the known data group we ought know the specific sequence of each nodes. And for two different structures calculated by $K 2$ which are $G_{B 1}$ and $G_{B 2}$, for:

$$
\frac{p\left(G_{B 1} \mid D\right)}{p\left(G_{B 2} \mid D\right)}=\frac{\frac{p\left(G_{B 1}, D\right)}{p(D)}}{\frac{p\left(G_{B 2}, D\right)}{P(D)}}=\frac{p\left(G_{B 1}, D\right)}{p\left(G_{B 2}, D\right)}
$$

We only need to compare each structure's posterior probability to pick best structure but should obey the four following assumptions:

(1) all the variable is scattered variable ;

(2)the data should obey to Dirichlet distribution;

(3)the data group is full without lost data ;

(4)priori probability $\pi(\theta)$;

Based on those four assumptions Cooper (1992) build the following formula:

$$
p\left(G_{B} \mid D\right)=p\left(G_{B}\right) p\left(D \mid G_{B}\right)=p\left(G_{B}\right) \cdot \prod_{i=1}^{n} \prod_{j=1}^{q_{i}} \frac{\left(r_{i}-1\right) !}{\left(N_{i j}+r_{i}-1\right)} \cdot \prod_{k=1}^{r_{i}} N_{i j k} !
$$

The $p\left(G_{B}\right)$ is the priori probability of structure and $\left\{x_{i}^{1}, \ldots, x_{j}^{r_{i}}\right\}$ is the range of $x_{i}$, the sequence of $\Pi_{i}$ is $1, \ldots, q_{i}$ and $N_{i j}=\sum_{k=1}^{r_{i}} N_{i j k}, N_{i j k}$ is the case amount of group $D$ which obey to the condition $X_{i}=x_{i}^{k}$ and $\pi_{i}=j^{[3]}$

\section{Monetary network construction}

Date preprocessing.

We collect the data form the website of China National Bureau of Statistics ${ }^{[4]}$ and the World Bank $^{[5]}$. The data contain 174 nodes of 13 years about 23 statistic groups, which are national economic accounting, international trade, finance and insurance, etc. And we use this formula $n_{i}=\left(n_{i}-\mu\right) / \sigma$ to standardize the data then we put the data into SPSS to draw statistic figure to make the data more objective from node1 to 8 .

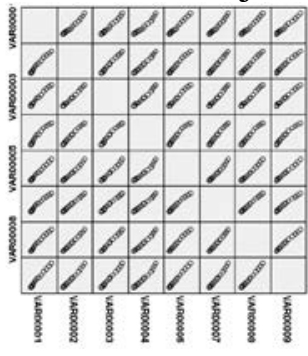

Fig. 1 Scattered point distribution

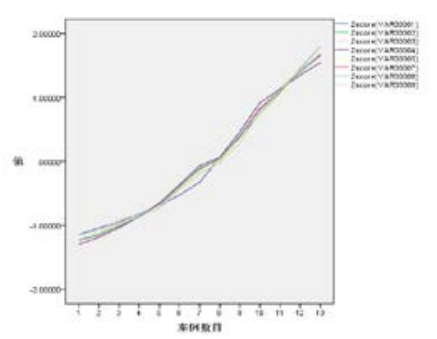

Fig. 2 The trend (standardized)

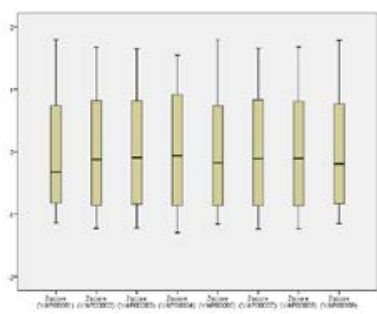

Fig. 3 Box fig.

\section{Using $K 2$ and MCMC to build network and Visualization.}

There are two very different approaches to structure learning: constraint-based and search-and-score. In the more popular search-and-score approach, we perform a search through the space of possible DAGs, and either return the best one found (a point estimate), or return a sample of the models found (an approximation to the Bayesian posterior). 
Unfortunately, the number of DAGs as a function of the number of nodes, $G(n)$, is super-exponential in n. A closed form formula for $G(n)$ is not known, but the first few values are shown below (from Cooper, 1999). ${ }^{[6]}$

The K2 algorithm (Cooper and Herskovits, 1992) is a greedy search algorithm that works as follows. Initially each node has no parents. It then adds incrementally that parent whose addition most increases the score of the resulting structure. When the addition of no single parent can increase the score, it stops adding parents to the node. Since we are using a fixed ordering, we do not need to check for cycles, and can choose the parents for each node independently. ${ }^{[7]}$

After running, the learn_structure_K2.m of the toolkit FullBNT-1.0.7 $7^{[8]}$ and we pick all the elements which equal to one out by this instruction: $[\mathrm{a}, \mathrm{b}]=$ find(Matrix==1). Finally we got 1775 lines, the figure displayed like this:

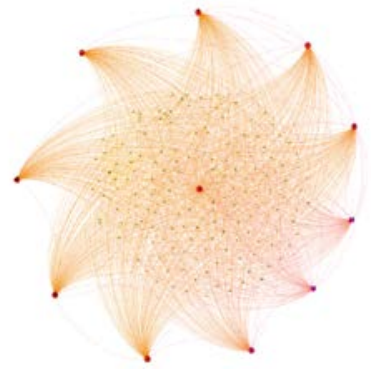

Fig.4 Monetary system (crook)

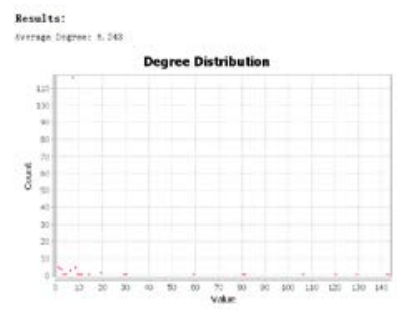

Fig. 7 Degree Distribution(K2)

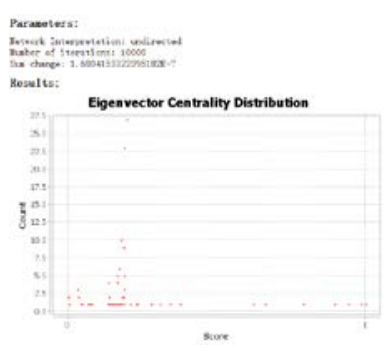

Fig. 5 ECD. (K2)

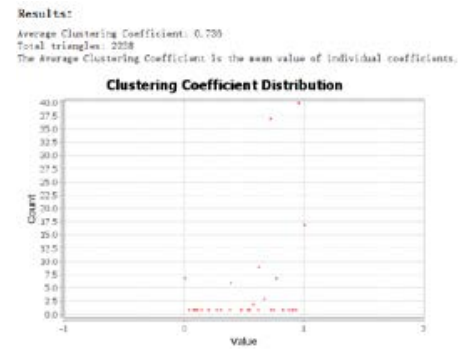

Fig. 8 CCD.(K2)

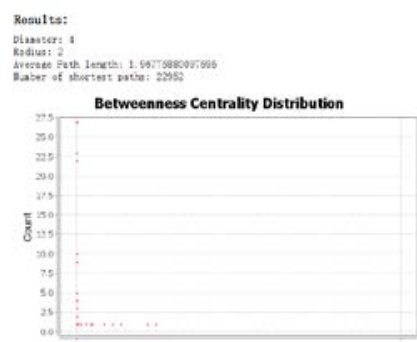

Fig. 6 BCD.(K2)

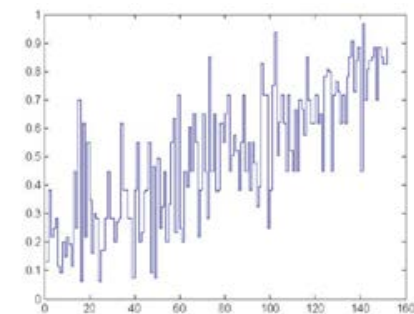

Fig.9 Node probability trend

In Fig. 4 we find that there have 11 nodes with most connections than the other, those nodes tell us during 2002 to 2014, they have remarkable influence to the macroeconomic. And we also analyze some basic information of the structure. The first picture is the Eigenvector centrality, which is a measure of the influence of a node in a network. It assigns relative scores to all nodes in the network based on the concept that connections to high-scoring nodes contribute more to the score of the node in question than equal connections to low-scoring nodes. ${ }^{[9]}$ In our net few nodes have most linking most nodes have few connections by using $K 2$ method. And the average centrality distribution is 1.96775 , the average degree is 6.243 the clustering coefficient distribution is 0.736 . We also use Matlab to draw the CPDs distribution the result is Fig. 9.

Unfortunately, the number of DAGs as a function of the number of nodes, $G(n)$, is super-exponential in n. A closed form formula for $G(n)$ is not known, but the first few values are shown below (from Cooper, 1999). ${ }^{[10]}$

As we montioned $K 2$ mothod is based on the known orger of each nodes which is $G(n)$, but in the most condition the sequence is unknown thus we choice another mothod which is MCMC. Markov Chain Monte Carlo (MCMC) algorithm called Metropolis-Hastings (MH) to search the space of all DAGs. The standard proposal distribution is to consider moving to all nearest neighbors in the sense defined above. The result is Fig.10, In the end we can also plot the acceptance ratio versus number of MCMC steps, as a crude convergence diagnostic by calculate 1000, 5000, 10000 times as Fig.14 shown and the answer is already equal to 2.5 . 


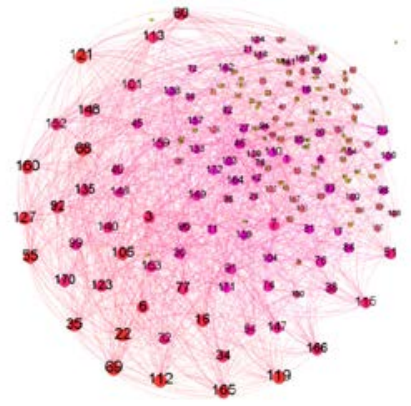

Fig. $10 \mathrm{MS}$ (MCMC)

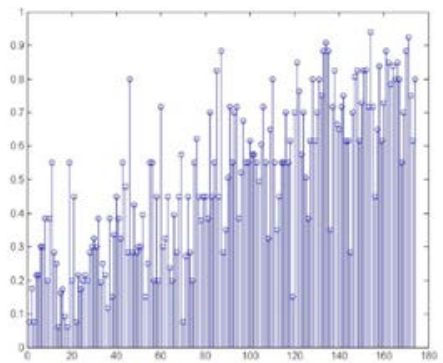

Fig. 13 Probability scatter diagram(MCMC)

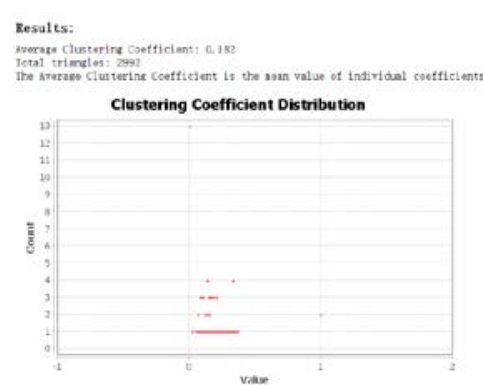

Fig. 12 CCD.(MCMC)

Fig. 11 DB.(MCMC)

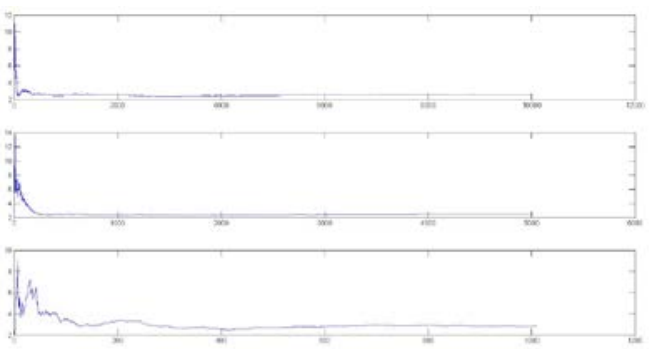

Fig. 14 the acceptance ratio

\section{Summary and Future Work}

In our network we find the main factors have more influence on the monetary system (N.M. is Near money;T.I.F.A. is Total investment in fixed assets, the result is :

Table 1 Main Factors

\begin{tabular}{|c|c|c|c|c|c|c|c|}
\hline Source & Target & Source & Target & Source & Target & Source & Target \\
\hline N.M. & T.I.F.A. & GDP & N.M. & K2 N.M. & State funds & $\begin{array}{c}\text { Rural } \\
\text { Consumption }\end{array}$ & N.M. \\
\hline N.M. & $\begin{array}{c}\text { Stock Exchange } \\
\text { Amount }\end{array}$ & Retail price index & N.M. & K2 N.M. & T.I.F.A. & output value & N.M. \\
\hline N.M. & Stock issue of SH S.E & $\begin{array}{c}\text { finance } \\
\text { expenditure }\end{array}$ & N.M. & K2 N.M. & Final goods & savings & N.M. \\
\hline N.M. & $\begin{array}{l}\text { Technological } \\
\text { Invention }\end{array}$ & tax revenue & N.M. & K2 N.M. & capital formation & Unemployment & N.M. \\
\hline K2 N.M. & GDP index & Sales Tax & N.M. & K2 N.M. & Exports & Education funds & N.M. \\
\hline K2 N.M. & GPD(per capita) & deposits & N.M. & K2 N.M. & Imports & R\&D science & N.M. \\
\hline K2 N.M. & GNI & M2 & N.M. & K2 N.M. & House construction & Public Libraries & N.M. \\
\hline K2 N.M. & $\begin{array}{l}\text { Households } \\
\text { consumption }\end{array}$ & gold reserve & N.M. & K2 N.M. & Total retail sales & K2 N.M. & $\begin{array}{l}\text { Stock } \\
\text { E. }\end{array}$ \\
\hline K2 N.M. & Rural Consumption & $\begin{array}{c}\text { Stock market } \\
\text { value }\end{array}$ & N.M. & K2 N.M. & $\$$ rate & K2 N.M. & $\begin{array}{l}\text { Stock } \\
\text { I. }\end{array}$ \\
\hline K2 N.M. & Urban Consumption & Futures turnover & N.M. & & & & \\
\hline
\end{tabular}

In future work we hope to continue our researching about macroeconomic and monetary system. We decide to use MCMC method to reveal the volatility of CPI and currency flowing amount. Also we hope to use software like Netica to form a warning system about CPI that can tell us when the society may face inflation and deflation.

\section{Reference}

[1] John Maynard Keynes. <The general theory of employment, interest and money>. Page 173

[2] John Maynard Keynes. < The general theory of employment, interest and money>. Page 34

[3] Wang Shuangcheng, <Bayesian Network Learning Infering and Applying>.Shang Hai.2010.2.page12 
[4] http://www.stats.gov.cn/

[5] http://data.worldbank.org/

[6] http://www.cs.ubc.ca/ murphyk/Software/BNT/usage_02nov13.html\#K2 ." How to use the Bayes Net Toolbox”(PDF)

[7] Gregory F. Cooper, Edward Herskovits. A Bayesian method for the induction of probabilistic networks from data. October 1992, Volume 9, Issue 4, pp 309-347

[9] K Murphy. The bayes net toolbox for matlab. Computing science and statistics, 2001

[10]CN Glymour, GF Cooper. Computation, causation, and discovery.1999 